\title{
Leishmania ITS1 Is Genetically Divergent in Asymptomatic and Symptomatic Visceral Leishmaniasis: Results of a Study in Southern Iran
}

\author{
Zahra Rezaei, ${ }^{1}$ Eqlima Azarang, ${ }^{2}$ Saeed Shahabi, ${ }^{2}$ Mostafa Omidian, \\ Bahman Pourabbas, ${ }^{1}$ and Bahador Sarkari $\left(^{2,3}\right.$ \\ ${ }^{1}$ Professor Alborzi Clinical Microbiology Research Center, Shiraz University of Medical Sciences, Shiraz, Iran \\ ${ }^{2}$ Department of Parasitology and Mycology, School of Medicine, Shiraz University of Medical Sciences, Shiraz, Iran \\ ${ }^{3}$ Basic Sciences in Infectious Diseases Research Center, Shiraz University of Medical Sciences, Shiraz, Iran
}

Correspondence should be addressed to Bahador Sarkari; sarkarib@sums.ac.ir

Received 8 June 2020; Accepted 13 July 2020; Published 28 August 2020

Academic Editor: Shyam Sundar

Copyright (C) 2020 Zahra Rezaei et al. This is an open access article distributed under the Creative Commons Attribution License, which permits unrestricted use, distribution, and reproduction in any medium, provided the original work is properly cited.

It has been documented that the genotypic traits in symptomatic and asymptomatic cases of visceral leishmaniasis (VL) may be different. The current study aimed to find out and compare the genotype and intraspecies diversity of Leishmania Internal Transcribed Spacer 1 (ITS1) from asymptomatic and symptomatic VL cases in southern Iran. Methods. Buffy coat samples from seven VL patients, with clinical signs and symptoms, and seven asymptomatic VL cases, were evaluated in this study. Samples of asymptomatic individuals were obtained from children living in a VL endemic area in southern Iran, while the samples of symptomatic subjects were obtained from patients admitted to hospitals with a diagnosis of VL. DNA was extracted from the buffy coats of the samples and PCR-amplified, targeting the ITS1of Leishmania. The PCR products were sequenced, and the consensus sequences were assembled and multiple-aligned with a set of Leishmania strains retrieved from the GenBank, using Clustal W. The phylogenetic tree was rooted, using MEGAX software, and the diversities based on haplotype and nucleotides, as well as the number of polymorphic sites, were measured using DnaSP v5.0 software. The results of ITS1 sequencing in 5 out of 7 asymptomatic VL cases showed $99.25 \%$ to $100 \%$ similarity with the Leishmania infantum ITS1 sequence (accessed number: MN648746), and one isolate was considered as just Leishmania sp. In one sample, $99.75 \%$ similarity was seen with the ITS1 sequence of Crithidia fasciculata. Of the symptomatic VL patients, the PCR product revealed a $340 \mathrm{bp}$ band corresponding to L. infantum in all of the samples. By analyzing the ITS1 sequences, all seven sequences formed a clade somewhat different from other Leishmania species and considered as Leishmania sp. Haplotype and nucleotide diversity were much more prevalent in symptomatic cases where six haplotypes were seen in the ITS1 of Leishmania from symptomatic patients and only two haplotypes were observed in the samples from asymptomatic cases. The findings of the current study showed that the Leishmania ITS1 from symptomatic VL and asymptomatic cases has significant genetic differences. Besides, infection with Crithidia fasciculata was reported, for the first time, in an asymptomatic case, which deserves further study.

\section{Introduction}

Leishmaniasis continues to be one of the health challenges in many countries of the world, with an estimated annual worldwide incidence of 700,000 to $1,300,000$ for cutaneous leishmaniasis (CL) and approximately 500,000 cases for visceral leishmaniasis (VL) $[1,2]$. The disease is caused by different species of Leishmania parasites and can produce a range of clinical symptoms based on the type of parasite involved $[3,4]$. The spectrum begins in a general category of localized or diffuse skin involvement, which is a self-limiting skin condition. Also, the parasite can cause skin-mucosal involvement and eventually cause systemic and fatal disease by involving the viscera and internal organs.

In $\mathrm{VL}$, most cases do not progress to overt disease and remain asymptomatic [5-7]. The proportion of 
asymptomatic to symptomatic VL cases has been reported to be $4: 1$ in Kenya, 11:1 in Sudan, and 9:1 in India and Nepal [8-10]. In asymptomatic VL cases, there are parasites in their peripheral blood circulation that can be detected by molecular methods or even culture [11-13]. Asymptomatic humans or animals may constitute a reservoir for VL and contribute a risk of distributing the VL to another human or animals [5, 13-16].

Although most of the seroconverted asymptomatic VL cases do not develop an acute form of the disease, however, it has been shown that the progression to the overt form of the disease is associated with high positive qPCR, as well as rK39 ELISA or DAT antibody titers [17]. Accordingly, it has been suggested that healthy people living in given VL endemic areas having high antibody titers or being qPCR positive have an increased chance to progress to VL disease [17]. qPCR on blood samples showed 500 times less parasitemia in asymptomatic cases in comparison with the symptomatic ones in India [12].

Several studies documented that the genotypic traits in symptomatic and asymptomatic cases of VL may vary $[18,19]$. A study in southern France showed a significant genetic difference between Leishmania species, isolated from asymptomatic and symptomatic patients, especially those with HIV/VL coinfection [18]. The study also revealed that the isolates from asymptomatic cases have a weak polymorphism in their parasitic genome [18].

Apart from the parasite genotype, the host's genetic background can be involved in creating asymptomatic or symptomatic forms of VL [20]. It has been shown that the symptomatic VL is associated with the gene encoding a receptor for transforming growth factor beta (TGF- $\beta$ ) whereas the asymptomatic trait is linked to the gene encoding IIa receptor for the Fc fragment of IgG [20]. However, the association between SNP/HLA genotyping and progression from asymptomatic or seroconversion to VL overt disease has been insignificant [17]. Yet, another study in Brazil reported an association between the VL phenotype with SNP on chromosome 9 and 19 [21].

The current study aimed to find out and compare the genotype and intraspecies diversity of Leishmania isolated from asymptomatic and symptomatic VL cases in southern Iran.

\section{Materials and Methods}

2.1. Study Subjects. The protocol of this study was approved by the Ethical Committee of Shiraz University of Medical Sciences (SUMS). Informed consent was obtained from the patients or their guardians in the case of children. The subjects of this study were two groups of people. The first group consisted of 7 children admitted to Nemazee Hospital, Shiraz University of Medical Sciences, Shiraz, Iran, with clinical signs and symptoms of the disease. These VL patients had more than two weeks of fever, had hepatosplenomegaly, and they were all positive in IFAT (titer $>64$ ), using an Iranian strain of L. infantum (MCAN/IR/14/M14) as an antigen and confirmed by a real-time PCR and rK39-rapid diagnostic test (Kalazar Detect ${ }^{\mathrm{TM}}$ Rapid Test for VL, InBios,
Seattle, USA). They all recovered after either antimonial or amphotericin B therapy. The second group consisted of seven asymptomatic children who lived in the Sarmahshed area of Kazeroun County, one of the main endemic foci of $\mathrm{VL}$ in the south of Iran $[13,22,23]$. The children included 4 girls and 3 boys, ranging in age from 1 to 12 years. These children were selected from those who had been confirmed for Leishmania infection in our previous study, by using the ELISA system using crude antigen of Iranian strain of L. infantum (MCAN/IR/14/M14) and molecular methods [13]. The presence of Leishmania infection in these children was confirmed by PCR, using ITS2 primers. These children had no clinical signs or symptoms of VL, had no previous history of the disease, and have not developed the overt disease after one year of follow-up. Also, there were not any active VL cases in the children's family members during sample collection.

2.2. DNA Extraction and Nested-PCR. For both groups of the subjects, DNA was extracted from the buffy coats of whole blood $(5 \mathrm{ml})$ of each subject, using a commercial kit. PCR was performed on the extracted DNA to amplify the ITS1 fragment, using LITSR (ITS1-F) CTGGATCATTTTCCGATG and L5-8S-(ITS1-R) TGATACCACTTATCGCACTT primers $[24,25]$. The PCR program was set as follows: initial denaturation at $95^{\circ} \mathrm{C}$ for $5 \mathrm{~min}$, followed by 30 cycles at $94^{\circ} \mathrm{C}$ for $30 \mathrm{~s}, 55^{\circ} \mathrm{C}$ for $45 \mathrm{~s}$, and $72^{\circ} \mathrm{C}$ for $1 \mathrm{~min}$ and a final extension at $72^{\circ} \mathrm{C}$ for $5 \mathrm{~min}$ in an Eppendorf thermocycler. PCR products were separated by electrophoresis on $1.5 \%$ agarose gel. The PCR products were sequenced bilaterally for the ITS1 genomic fragment, using the same pair of primers used in the PCR assay.

2.3. Phylogenetic and Genetic Analysis. Chromas program, as implemented in the software BioEdit version 7.2.5, was used to view and analyze the nucleotide sequences [26]. Consensus sequences were assembled and multiple-aligned with a set of Leishmania strains retrieved from the GenBank, using Clustal W implemented in the above mentioned software [26]. The final aligned sequences with a total of 344 positions were converted in the FASTA and MEGA format for further analyse by using MEGAX software [27]. Phylogenetic relationships were reconstructed, using a Bayesian inference (BI) tree in MRBAYES, version 3.1.2 [28]. The Bayesian inference was performed with two simultaneous runs and four search chains within each run (three heated chains and one cold chain) for 10,000,000 generations, sampling trees every 1000 generations using the Markov chain Monte Carlo method. In our analysis, we left the prior on-state frequencies at its default setting (prset statefreqpr $=$ Dirichlet $(1,1,1,1))$. The structure of the substitution model was determined by setting of lset $n s t=6$ and rates $=$ equal (flat Dirichlet $(1,1,1,1,1,1)$ distribution). The default appropriate setting of the prior for the proportion of invariable sites was set with a uniform distribution between 0 and 1. The prior for shape of scaled gamma distribution of site rates and also for the proportion of invariable sites was considered as uniform. For topology, the prior was 
considered as an equal probability on all distinct, and for a partition-specific rate multiplier, it was fixed (1.0).

The reliability of nodes was assessed using the Bayesian posterior probability for Bayesian analysis. Trees were rooted with the sequence of Crithidia fasciculata (Accession no. MT302171). Genetic diversity based on haplotype diversity (Hd) and nucleotide diversity $(\pi)$, as well as the number of polymorphic sites, were measured, using DnaSP v5.0 software.

\section{Results}

Electrophoresis of the PCR product of ITS1 fragments of Leishmania isolates from asymptomatic subjects revealed a $340 \mathrm{bp}$ band in six samples corresponding to L. infantum (Figure 1). DNA of L. infantum and L. tropica were used as the positive controls. ITS1 sequencing results for the 7 asymptomatic cases showed that 5 of the samples had $100 \%$ similarity to the ITS1 sequence of $L$. infantum, with an accession no. MN648746, registered in the GenBank (lanes 1, 3, 4, 5 and 6 in Figure 1). One of the samples was similar to the ITS1 sequence of the Leishmania genome (lane 7 in Figure 1). The most surprising one was the sequencing result of one of the samples (lane 2 in Figure 1, about $410 \mathrm{bp}$ ) which had $99.75 \%$ similarity with the ITS1 sequence of the parasite Crithidia fasciculata (accession no. HM004585).

Of the VL patients, electrophoresis of the PCR product revealed a $340 \mathrm{bp}$ band corresponding to L. infantum in all of the samples (Figure 2).

3.1. Phylogenetic Analysis. The nucleotide sequences generated in this study have been deposited in GenBank under accession no. MT302158-71. All seven Leishmania sequences isolated from symptomatic patients along with one Leishmania sequence isolated from an asymptomatic patient formed a clade somewhat different from other Leishmania species and considered as Leishmania sp. (Figure 3). Monophylogeny of these two clades was supported with high Bayesian posterior probability (Figure 1). A sequence of Crithidia (accession no. MT302171), isolated from an asymptomatic patient, was closely related with a sequence of Crithidia fasciculata with accession no. HM004585 (Figure 3).

3.2. Genetic Diversity. Based on the sequences of the ITS1 fragment of the isolates, 29 sites were polymorphic and 28 sites were parsimony informative, resulting in the identification of 8 haplotypes. From 28 parsimony informative sites, 27 sites had two variants and one had three variants. The number of parsimony informative sites with four variants was 3 . Of the 8 observed haplotypes, 2 haplotypes were seen in the Leishmania sequences isolated from asymptomatic patients and six haplotypes were seen in Leishmania sequences isolated from symptomatic patients. No haplotype was shared between these two groups. Haplotype diversity (Hd) and nucleotide diversity $(\pi)$ values were 0.952 and 0.059 in Leishmania sequences

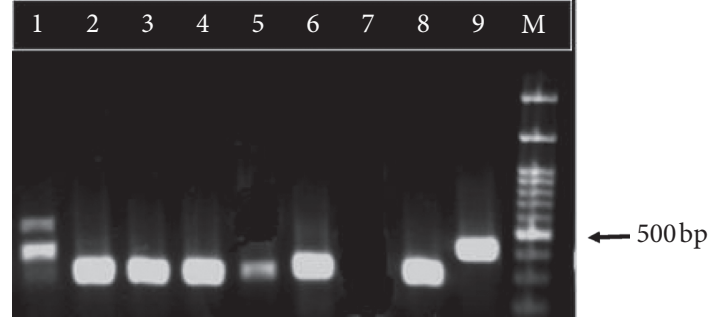

FIGURE 1: PCR product electrophoresis for ITS1 fragments of Leishmania from asymptomatic children on $1.5 \%$ agarose gel. Lane 1-6: isolated samples from asymptomatic children; lane 7: negative control; lane 8: L. infantum positive control; lane 9: L. tropica positive control; and lane M: $1 \mathrm{~kb}$ molecular weight marker.

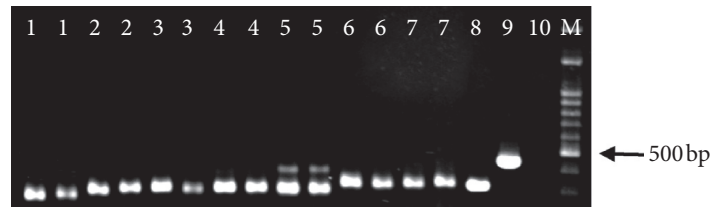

FIGURE 2: PCR product electrophoresis for ITS1 fragments of Leishmania from VL patients on 1.5\% agarose gel. Lane 1-7 (in duplicate): Leishmania isolated from VL patients; 8: L. infantum positive control; 9: L. tropica positive control; 10: negative control; and $\mathrm{M}: 1 \mathrm{~kb}$ molecular weight marker.

isolated from symptomatic patients, respectively, while these two diversity values ( $\mathrm{Hd}$ and $\pi$ ) were 0.33 and 0.047 for Leishmania sequences isolated from asymptomatic patients. Haplotype and nucleotide diversity in the ITS1 fragment of Leishmania were much more prevalent in symptomatic in comparison with asymptomatic cases. The alignment of the ITS1 sequences in the current study is shown in Figure 4.

\section{Discussion}

For many years, there has been speculation that, in VL, the Leishmania genotypic differences involve in asymptomatic or symptomatic forms of the disease. In the present study, we used the ITS1 fragment to analyze the genetic diversity of Leishmania isolates in people infected with Leishmania but without clinical signs or symptoms and those symptomatic VL patients.

A comparison of Leishmania ITS1 sequences from asymptomatic cases revealed that the sequences are mainly the same with low polymorphism. This is maybe because these genotypes are derived from the same clone of the parasite circulating in the areas that have not had the opportunity to differentiate and find differences. It may also be due to the pressure of the host's immune system, which prevents the parasite from differentiating and keeps it in a nonpathogenic state [18]. The findings of the current study are consistent with those of a previous study in France which reported a very weak genetic diversity in Leishmania isolates from asymptomatic cases in comparison with isolates from the VL patients [18]. In the present study, six haplotypes 


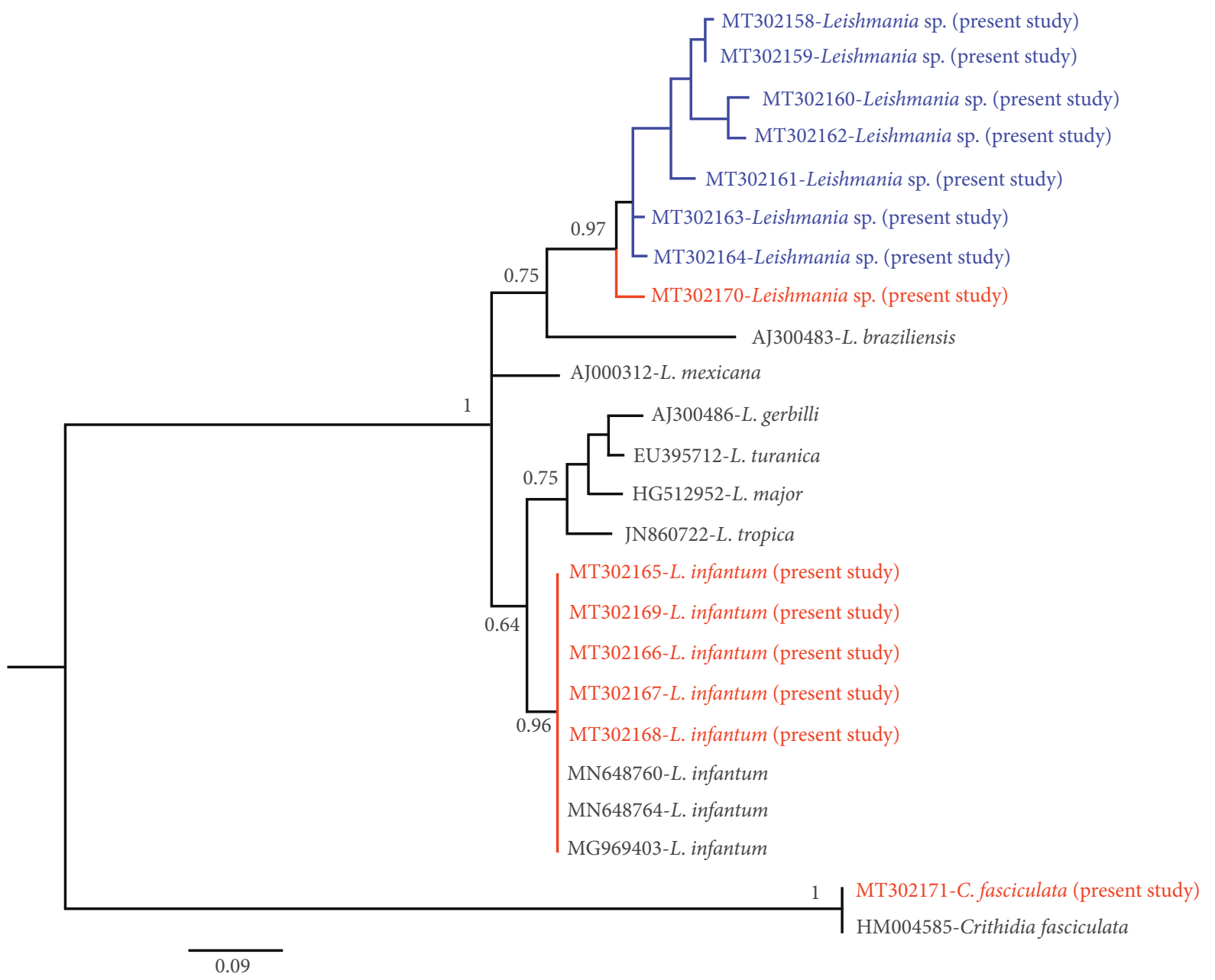

Figure 3: Phylogenetic tree of representative sequences of Leishmania from asymptomatic and symptomatic VL cases and reference sequences, based on the ITS1 fragment. Posterior probability values from the Bayesian analysis for nodes are indicated below or above branches. Samples isolated from asymptomatic and symptomatic cases are shown in red and blue color, respectively.

were seen in symptomatic cases whereas only two haplotypes were observed in the ITS1 of Leishmania from asymptomatic cases. This finding again is consistent with the findings of Hide et al. in France who found fewer haplotypes in asymptomatic isolates in comparison with the isolates from VL disease [18].

In one of the asymptomatic cases, infection with C. fasciculata was detected. Since 1980, coinfections of Leishmania with apparently single-host trypanosomatids have been reported, some of which in individuals with healthy immune systems $[29,30]$. Recently, in a study by Ghabakhloo et al., in Fars Province, where the current study was carried out, C. fasciculata was detected in $1.8 \%$ of CL patients [31]. The interesting point in their study was that, in patients' lesion, only C. fasciculata has been identified and Leishmania parasites have not been found. Therefore, the coinfection of Leishmania and C. fasciculata has not been the case, and CL lesion has been attributed to C. fasciculate [31]. Also, a recent study in Brazil reported Crithidia-related parasites in two fatal VL-like diseases, both of which have been resistant to current disease treatments [32]. The importance of our finding is that the presence of Crithidia in people living in endemic areas of VL may cause a cross reaction in serological tests and lead to false-positive results. Previous studies have confirmed the cross reaction of Crithidia with Leishmania in serological tests such as ELISA and IFA [33]. Besides, as a result of this cross reaction, VL-related seroepidemiological studies may show a higher prevalence in such areas. Although Crithidia or Crithidia-like protozoa have been reported in patients with CL or VL, the role of this protozoan in causing disease in humans has so far been in a state of ambiguity and requires extensive studies in this field.

In the current study, variation was seen in Leishmania ITS1 sequences in VL patients, so that even in two patients, the same sequences were not seen, and all had significant differences with each other. Previous studies have also reported similar diversity for Leishmania isolated from VL patients in comparison with asymptomatic cases [18, 34]. It should not be forgotten that all of the asymptomatic cases in this study were from the same area, whereas the VL patients were from different areas of Fars 


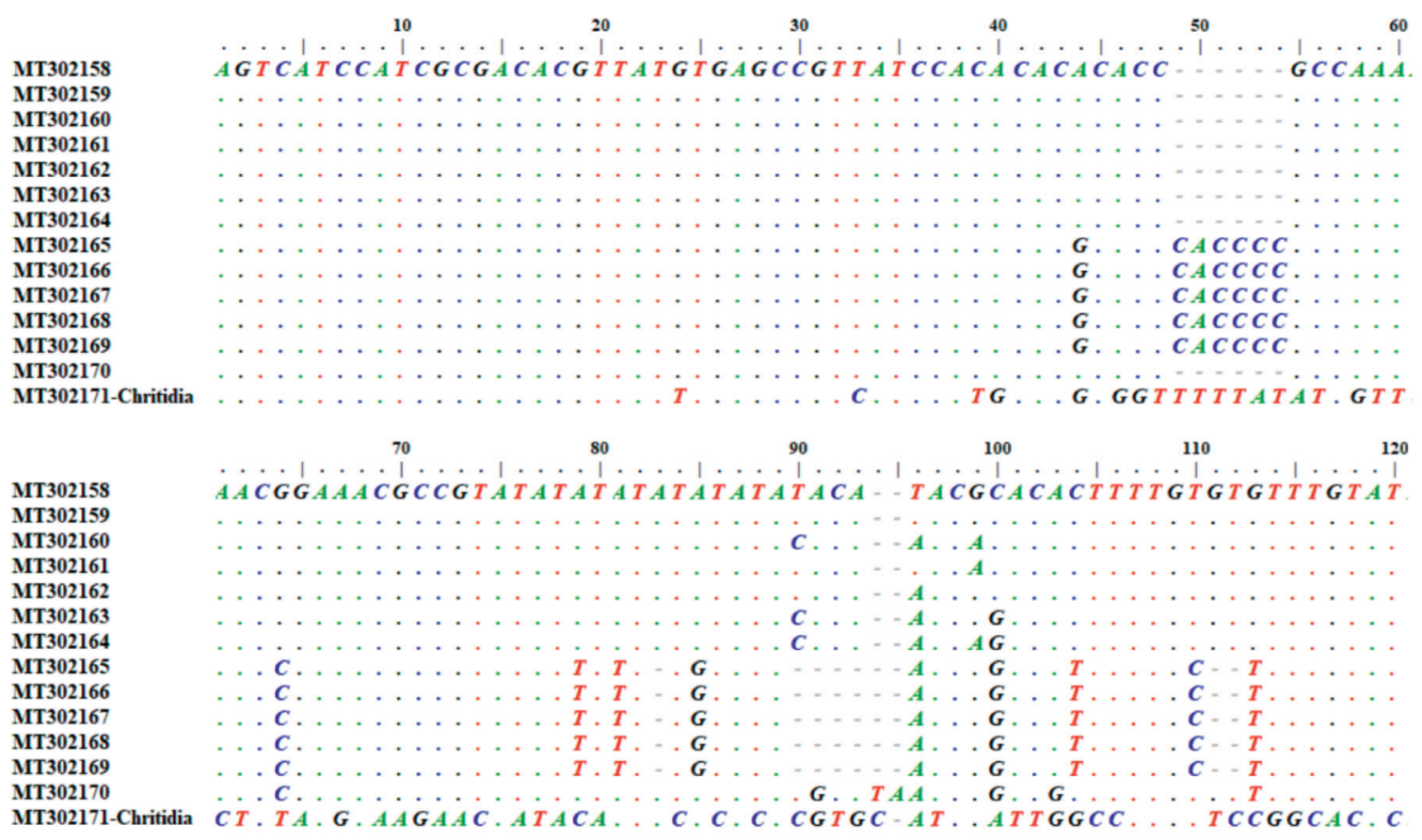

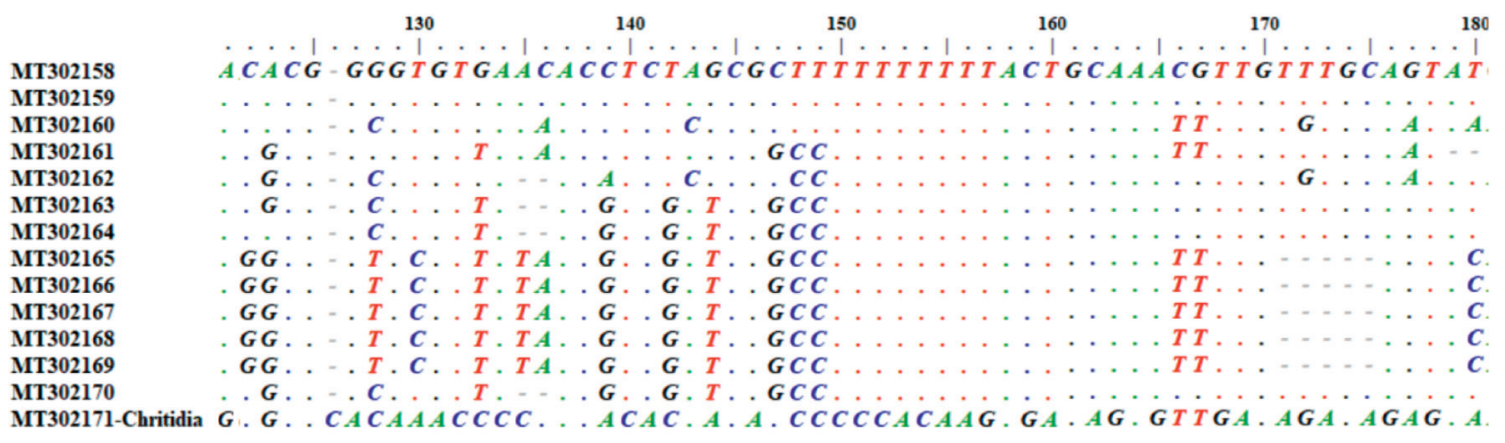

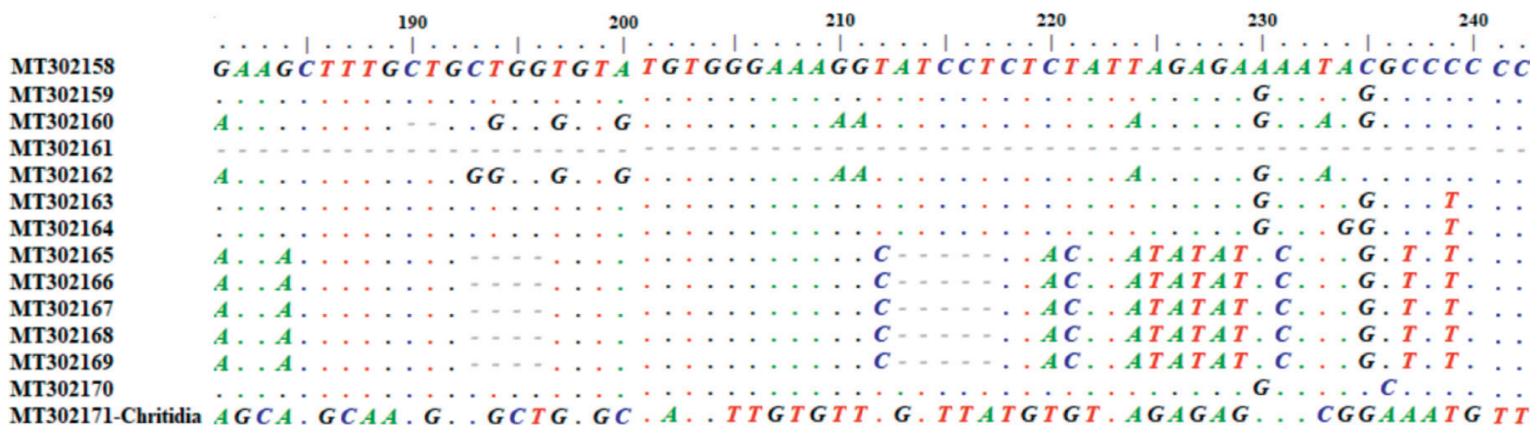

FIgURE 4: Alignments of ITS1 sequences of Leishmania from asymptomatic and symptomatic VL cases.

Province in the south of Iran. It is, therefore, recommended that asymptomatic VL cases from other areas be evaluated to see whether such homogeny exists between the isolates from different geographical regions. The main limitations of the study were the low numbers of both VL and asymptomatic cases; therefore, studying a larger population will provide robust conclusions. Another limitation of the study was the presence of VL patients from different endemic areas, while the asymptomatic individuals were all from the same area.

\section{Conclusions}

Findings of the current study showed that Leishmania isolated from symptomatic and asymptomatic VL cases has significant genetic diversity in the ITS1 fragment. The study also revealed a weak polymorphism in the ITS1 of Leishmania from asymptomatic cases, in comparison with those isolated from VL patients. Besides, infection with Crithidia fasciculata was reported, for the first time, in an asymptomatic VL case, which deserves further study. 


\section{Data Availability}

The data used to support the findings of this study are included within the article.

\section{Disclosure}

This study was the subject of the MD dissertation of Dr. Eqlima Azarang.

\section{Conflicts of Interest}

The authors declare no conflicts of interest.

\section{Authors' Contributions}

BS, ZR, and BP designed the study. EA, MO, and SS involved in data collection and data analysis. BS and ZR prepared the manuscript draft. All authors read and approved the final version of the manuscript.

\section{Acknowledgments}

This study was financially supported by the office of vicechancellor for research of Shiraz University of Medical Sciences (Grant no. 1396-01-01-16111).

\section{References}

[1] I. Okwor and J. Uzonna, "Social and economic burden of human leishmaniasis," The American Journal of Tropical Medicine and Hygiene, vol. 94, no. 3, pp. 489-493, 2016.

[2] D. Pace, "Leishmaniasis," Journal of Infection, vol. 69, no. Suppl 1, pp. S10-S18, 2014.

[3] B. Sarkari, N. B Ahmadpour, M. H Motazedian et al., "Interand intraspecific variations of Leishmania strains isolated from patients with cutaneous and visceral leishmaniases in Fars province, south of Iran," Iranian Journal of Medical Sciences, vol. 41, no. 3, pp. 209-216, 2016.

[4] J. A. R. Postigo, "Leishmaniasis in the world health organization eastern mediterranean region," International Journal of Antimicrobial Agents, vol. 36, no. Suppl 1, pp. S62-S65, 2010.

[5] A. Alborzi, F. Shahian, B. Pourabbas, M. Ziyaeyan, G. R. Pouladfar, and J. Mardaneh, "Detection of Leishmania infantum kinetoplast DNA in the whole blood of asymptomatic individuals by PCR-ELISA and comparison with other infection markers in endemic areas, southern Iran," The American Journal of Tropical Medicine and Hygiene, vol. 79, no. 6, pp. 839-842, 2008.

[6] C. Riera, R. Fisa, P. Lpez-Chejade et al., "Asymptomatic infection byLeishmania infantumin blood donors from the Balearic Islands (Spain)," Transfusion, vol. 48, no. 7, pp. 1383-1389, 2008.

[7] B. Sarkari, F. Gadami, R. Shafiei et al., "Seroprevalence of Leishmania infection among the healthy blood donors in kalaazar endemic areas of Iran," Journal of Parasitic Diseases, vol. 39, no. 3, pp. 545-549, 2015.

[8] K. U. Schaefer, G. J. Schoone, G. S. Gachihi, A. S. Muller, P. A. Kager, and S. E. O. Meredith, "Visceral leishmaniasis: use of the polymerase chain reaction in an epidemiological study in Baringo District, Kenya," Transactions of the Royal Society of Tropical Medicine and Hygiene, vol. 89, no. 5, pp. 492-495, 1995.
[9] E. E. Zijlstra, A. Ismael, H. W. Ghalib, and A. M. El-Hassan, "Endemic kala-azar in eastern Sudan: a longitudinal study on the incidence of clinical and subclinical infection and postkala-azar dermal leishmaniasis," The American Journal of Tropical Medicine and Hygiene, vol. 51, no. 6, pp. 826-836, 1994.

[10] B. Ostyn, K. Gidwani, B. Khanal et al., "Incidence of symptomatic and asymptomatic Leishmania donovani infections in high-endemic foci in India and Nepal: a prospective study," PLoS Neglected Tropical Diseases, vol. 5, no. 10, p. e1284, 2011.

[11] P. Srivastava, K. Gidwani, A. Picado et al., "Molecular and serological markers ofLeishmania donovaniinfection in healthy individuals from endemic areas of Bihar, India," Tropical Medicine \& International Health, vol. 18, no. 5, pp. 548-554, 2013.

[12] M. Sudarshan and S. Sundar, "Parasite load estimation by qPCR differentiates between asymptomatic and symptomatic infection in Indian visceral leishmaniasis," Diagnostic Microbiology and Infectious Disease, vol. 80, no. 1, pp. 40-42, 2014.

[13] A. Layegh Gigloo, S. Bahador, R. Zahra, R. H. Gholam, and H. D. Mohammad, "Asymptomatic Leishmania infected children: a seroprevalence and molecular survey in a rural area of fars province, southern Iran," Journal of Tropical Medicine, vol. 2018, Article ID 8167247, 6 pages, 2018.

[14] S. Das, G. Matlashewski, G. S. Bhunia, S. Kesari, and P. Das, "Asymptomatic Leishmania infections in northern India: a threat for the elimination programme?," Transactions of the Royal Society of Tropical Medicine and Hygiene, vol. 108, no. 11, pp. 679-684, 2014.

[15] B. Mohammadi-Ghalehbin, R. H. Gholam, and S. Bahador, "A Leishmania infantum FML-ELISA for the detection of symptomatic and asymptomatic canine visceral leishmaniasis in an endemic area of Iran," Iranian Journal of Immunology, vol. 8, no. 4, pp. 244-250, 2011.

[16] A. Moshfe, M. Mohebali, G. Edrissian et al., "Canine visceral leishmaniasis: asymptomatic infected dogs as a source of L. infantum infection," Acta Tropica, vol. 112, no. 2, pp. 101-105, 2009.

[17] J. Chakravarty, H. Epco, K. Sangeeta et al., "Determinants for progression from asymptomatic infection to symptomatic visceral leishmaniasis: a cohort study," PLOS Neglected Tropical Diseases, vol. 13, no. 3, Article ID e0007216, 2019.

[18] M. Hide, E. Marion, C. Pomares, R. Fisa, P. Marty, and A. L. Bañuls, "Parasitic genotypes appear to differ in leishmaniasis patients compared with asymptomatic related carriers," International Journal for Parasitology, vol. 43, no. 5, pp. 389-397, 2013.

[19] L. Montoya, R. Piarroux, B. Gavignet et al., "Application of microsatellite genotyping to the study of a restricted Leishmania infantum focus: different genotype compositions in isolates from dogs and sand flies," The American Journal of Tropical Medicine and Hygiene, vol. 76, no. 5, pp. 888-895, 2007.

[20] J. L. Weirather, P. Duggal, E. L. Nascimento et al., "Comprehensive candidate gene analysis for symptomatic or asymptomatic outcomes ofLeishmania infantuminfection in Brazil," Annals of Human Genetics, vol. 81, no. 1, pp. 41-48, 2017.

[21] J. L. Weirather, P. Duggal, E. L. Nascimento et al., "Fine mapping under linkage peaks for symptomatic or asymptomatic outcomes of Leishmania infantum infection in Brazil," Infection, Genetics and Evolution, vol. 43, pp. 1-5, 2016.

[22] B. Sarkari, G. Hatam, and M. Ghatee, "Epidemiological features of visceral leishmaniasis in Fars province, southern 
Iran," Iranian Journal of Public Health, vol. 41, no. 4, pp. 94-99, 2012.

[23] B. Sarkari, "Visceral Leishmaniasis in southwestern Iran: a retrospective clinico-hematological analysis of 380 consecutive hospitalized cases (1999-2014)," PLoS One, vol. 11, no. 3, Article ID e0150406, 2016.

[24] N. O. el Tai, O. F. Osman, M. el Fari, W. Presber, and G. Schönian, "Genetic heterogeneity of ribosomal internal transcribed spacer in clinical samples of Leishmania donovani spotted on filter paper as revealed by single-strand conformation polymorphisms and sequencing," Transactions of the Royal Society of Tropical Medicine and Hygiene, vol. 94, no. 5, pp. $575-579,2000$.

[25] M. H. Davami, M. H. Motazedian, and B. Sarkari, "The changing profile of cutaneous leishmaniasis in a focus of the disease in Jahrom district, southern Iran," Annals of Tropical Medicine \& Parasitology, vol. 104, no. 5, pp. 377-82, 2010.

[26] T. A. Hall, Bioedit: A User-Friendly Biological Sequence Alignment Editor and Analysis Program for Windows 95/98/ Nt, no. c1979-c2000, pp. 95-98, no. Information Retrieval Ltd., London, UK, 1999.

[27] S. Kumar, G. Stecher, M. Li, C. Knyaz, and K. Tamura, "MEGA X: molecular evolutionary genetics analysis across computing platforms," Molecular Biology and Evolution, vol. 35, no. 6, pp. 1547-1549, 2018.

[28] F. Ronquist and J. P. Huelsenbeck, "MrBayes 3: Bayesian phylogenetic inference under mixed models," Bioinformatics, vol. 19, no. 12, pp. 1572-1574, 2003.

[29] G. Eslami, R Salehi, S Khosravi, and M Doudi, "Genetic analysis of clinical isolates of Leishmania major from Isfahan, Iran," Journal of Vector Borne Diseases, vol. 49, no. 3, pp. 168-174, 2012.

[30] A. Mirzapour, B. Alireza, and A. Marzieh, "Super infection of cutaneous leishmaniasis caused by Leishmania major and L. Tropica to Crithidia fasciculata in Shiraz, Iran," Iranian Journal of Public Health, vol. 48, no. 12, pp. 2285-2292, 2019.

[31] N. Ghobakhloo, M. H Motazedian, S Naderi, and S Ebrahimi, "Isolation of Crithidia spp. from lesions of immunocompetent patients with suspected cutaneous leishmaniasis in Iran," Tropical Medicine \&amp; International Health: TM \&amp; $I H$, vol. 24, no. 1, pp. 116-126, 2019.

[32] S. R. Maruyama, A. K. M. de Santana, N. T. Takamiya et al., "Non-leishmania parasite in fatal visceral leishmaniasis-like disease, Brazil," Emerging Infectious Diseases, vol. 25, no. 11, p. 2088, 2019.

[33] F. Martinkovic and A. Marinculic, "Antibodies against Leishmania cross-react with Crithidia luciliae: indirect immunofluorescence and Dot-ELISA study in dogs," Parasitology Research, vol. 98, no. 4, pp. 378-380, 2006.

[34] A. L. Bañuls, P. Bastien, C. Pomares, J. Arevalo, R. Fisa, and M. Hide, "Clinical pleiomorphism in human leishmaniases, with special mention of asymptomatic infection," Clinical Microbiology and Infection, vol. 17, no. 10, pp. 1451-1461, 2011. 